\title{
The Ring of Fire - An Internal Illumination System for Detector Sensitivity and Filter Bandpass Characterization
}

\author{
Victor E. Scarpine* ${ }^{\mathrm{a}}$, Stephen M. Kent ${ }^{\mathrm{a}}$, Susana E. Deustua ${ }^{\mathrm{b}}$, Michael J. Sholl ${ }^{\mathrm{c}}$, Stuart L. Mufson ${ }^{\mathrm{d}}$, \\ Melanie N. Ott ${ }^{\mathrm{e}}$, Matthew P. Wiesner, Brian J. Baptista ${ }^{\mathrm{d}}$ \\ ${ }^{a}$ Fermi National Accelerator Laboratory, P.O. Box 500, Batavia, IL, USA 60510; \\ ${ }^{\mathrm{b}}$ Space Telescope Science Institute, 3700 San Martin Dr., Baltimore, MD 21218; \\ 'Lawrence Berkeley National Laboratory, 1 Cyclotron Rd., Berkeley, CA 94720; \\ ${ }^{\mathrm{d}}$ Astronomy Dept., Indiana Univ. 727 E. Third St., Bloomington, IN 47405; \\ 'NASA Goddard Space Flight Center, Greenbelt, MD 20771; \\ ${ }^{\mathrm{f}}$ Dept. of Physics, Northern Illinois Univ. DeKalb, IL 60115
}

\begin{abstract}
We describe a prototype of an illumination system, the Ring of Fire (ROF), which is used as part of an internal calibration system for large focal plane detector arrays in TMA (Three Mirror Anastigmat) telescope designs. Such designs have been proposed for the SNAP (SuperNova Acceleration Probe) version of a Joint Dark Energy Mission (JDEM). The ROF system illuminates the focal plane with a light beam the closely matches that of the telescope and is used for creating high spatial frequency flat fields and monitoring filter bandpasses for experiments that demand a highly accurate characterization of the detectors. We present measurements of a mockup of this prototype ROF design including studies in variations in illumination across a large focal plane.
\end{abstract}

Keywords: Calibration, Telescope, Illumination

\section{INTRODUCTION}

A goal of future space missions such as the proposed Joint Dark Energy Mission (JDEM) [1] is to explore the nature of Dark Energy to unprecedented precision. Dark Energy manifests itself as an acceleration in the expansion rate of the universe today. Measuring the acceleration well enough to meaningfully constrain interesting physical models of Dark Energy requires improvements of order a magnitude in accuracy and precision of astronomical data beyond current programs. Most of the methods proposed to measure the Dark Energy equation of state involve surveying large areas of sky with both imaging and spectroscopy. Such surveys will require telescopes with large focal planes and a combination of a large number of optical and near-infrared imagers and long-slit spectrographs. Constraining sources of systematic uncertainties is key to distinguishing amongst the families of cosmological models as their differences in observational parameters are at the few percent level.

While space-based experiments have the advantage of significantly constraining systematic uncertainties for all the proposed Dark Energy probes arising from, for example, limitations due to the Earth's atmosphere, they must be able to constrain in addition, calibration uncertainties. Part of a well-developed calibration error budget is understanding and characterizing on-board hardware (detectors, filters, spectrographs, etc) not only before launch but also during the entire period of operation. An important component of accurate photometric calibration is the precise determination of the zero-points, a key aspect of which is having flat fields across the focal plane that are known to better than $1 \%$. The challenge for SNAP and proposed JDEM projects is that the relatively large active focal areas require creative solutions to the traditional methods of on-board flat fielding.

*scarpine@fnal.gov; phone 1630 840-2571 
The observatory calibration can be separated into instrumentation and astronomical components. Examples of instrument level calibration are characterization of detector linearity and stability, wavelength response of the optical elements, and shutter behavior. Astronomical calibration includes absolute color calibration, spectrophotometric flux standards, photometric zeropoint for each filter/detector combination, and flat fielding. Both components contribute to the photometric (and spectrophotometric) measurements (and error budgets) of science targets. Thus, for SNAP to ensure accurate and precise photometry of supernovae, both the instrumental and astronomical contributions to the calibration must be well determined. Science requirements on the dark energy properties correspond to uncertainties on the dark energy equation of state parameters $\mathrm{w}_{0}$ to 0.05 and $\mathrm{w}_{\mathrm{a}}$ to 0.3 or better, $\left(\mathrm{w}=\mathrm{w}_{0}+\mathrm{w}_{\mathrm{a}}(1-\mathrm{a}), \mathrm{a}=1 /(1+\mathrm{z}), \mathrm{z}\right.$ is the redshift) and in turn demand that the SNe Ia color calibration be $2 \%$ or better in the optical and better than $3 \%$ in the near infrared. Corresponding constraints on the photometry of weakly lensed galaxies are less stringent. This level of calibration is challenging, but not impossible.

One key factor in calibration is how well the pixel-to-pixel variations and the overall illumination patterns are known. Collectively these are referred to as flat fields, and should be known to better than $1 \%$ across the entire active focal area. Monitoring changes in filter transmission is critical if the photometric error budget is to be maintained throughout the lifetime of the mission. In order to characterize and monitor filter and detector behavior a mission will use an internal illumination system.

One attractive telescope design for a dark energy mission is the three-mirror anastigmat (TMA). Such a design has a wide field of view (greater than one degree on the sky), an intermediate focal plane, and an accessible exit pupil. One feature of such designs, however, is that the angle of incidence on the focal plane varies across the field, and if one introduces interference filters into the optical path, each filter will have a slightly different transmission function depending on its location on the focal plane. Interference filters have angle dependent transmission, and therefore a filter calibration system must mimic the range of incident photon angles on the focal plane. The characterization of filter transmission function with incidence angle is ideally carried out using a space-based system as well as a ground-based one.

The signal in each pixel of a detector is proportional to the flux incident on the telescope, the filter transmission, the device quantum efficiency and pixel size. All are a function of wavelength and spatial position. This pixel-to-pixel variation is corrected using flat field images. Once the calibration procedures have been applied, target objects can be photometered, measuring their correct relative brightness in the system defined by the effective instrumental bandpass (optics+instrument+detector) for both the imager and the spectrograph. Using this information for science requires converting these relative fluxes onto an absolute system. This procedure involves determining a zero-point for each bandpass, as well as a term proportional to the color of each object to allow for differences between standard and instrumental band passes. We note that the variation in filter transmission due to the incidence angle effects is commonly ignored in many narrow field detectors but must be accounted for in a wide field instrument.

Astronomical observations acquired with photoelectric detectors (e.g. CCDs, MCT, etc) correct for both the pixel-topixel variations due to gain or QE differences (high frequency) and for illumination differences across the detector (low frequency) by using flat fields. Using a light source that illuminates every pixel of the detector uniformly and that is spectrally identical to the target image creates an ideal flat field. Because the pixel response is spectrally sensitive flat fields are acquired through each filter or dispersing element (grism, prism, grating, etc). Dividing the flat field into the object image then flattens the pixel response. In practice, though, uniform illumination of a focal plane is hard to achieve, especially for large area focal surfaces.

There are several methods to obtaining flat fields for space applications:

- L-Flat - Low spatial frequency flats. Space telescopes typically use zodiacal light.

- P-Flat - High frequency flats, pixel-to-pixel variations. Space telescopes will point to the bright Earth, Jupiter or other similarly bright object.

- Sky Flats - Created by median filtering the target observations taken during the night.

- Star Flats - Obtained through repeated observations of dense star clusters

- Projection Flats - These are flats illuminated by a projected light source. They are used to flatten the high frequency variation, typically for spectral data, and have the disadvantage of not following the entire optical 
path as the lamps are placed after the primary and secondary mirrors. They are not used to correct the low frequency variation.

Ideally, a space mission will rely on a combination of projection flats, such as the Ring of Fire, and sky and star flats. Projects that require accurate absolute photometric calibration over the entire active imaging area, as in the case of JDEM, place stringent requirements on the flat fields, and most importantly on the control of systematic uncertainties. Pixel-to-pixel and size variations, if uncorrelated over the field of the detector, contribute statistically to the error budget. However, offsets between filter plus detector, e.g. several B filter plus CCDs, if not properly taken into account or characterized will contribute to the systematic uncertainty.

\section{RING OF FIRE ILLUMINATION PROJECTION SYSTEM}

The ROF functions as the diffuse source of irradiance [2]. The ROF places a diffuser in an annular ring around and just outside the exit pupil of the telescope beam. The diffuser illuminates the focal plane with a beam pattern that closely matches that of the telescope itself. The diffuser is illuminated by a set of optical fibers and a mirror system fed by light from integrating sphere or other illumination source that can be powered by a variety of lamps. Because the diffuser is outside the telescope beam, it does not require or rely on the presence of a shutter, and it can be used to add light to an existing astronomical scene such as has been one with the Hubble Space Telescope NICMOS detector in order to calibrated wavelength-dependent nonlinearitities (the Bohlin effect [3]). Figure 1 illustrates a cross-sectional view of an earlier version of SNAP proposed for JDEM, which shows the TMA design and exit pupil at the top of the light shield.

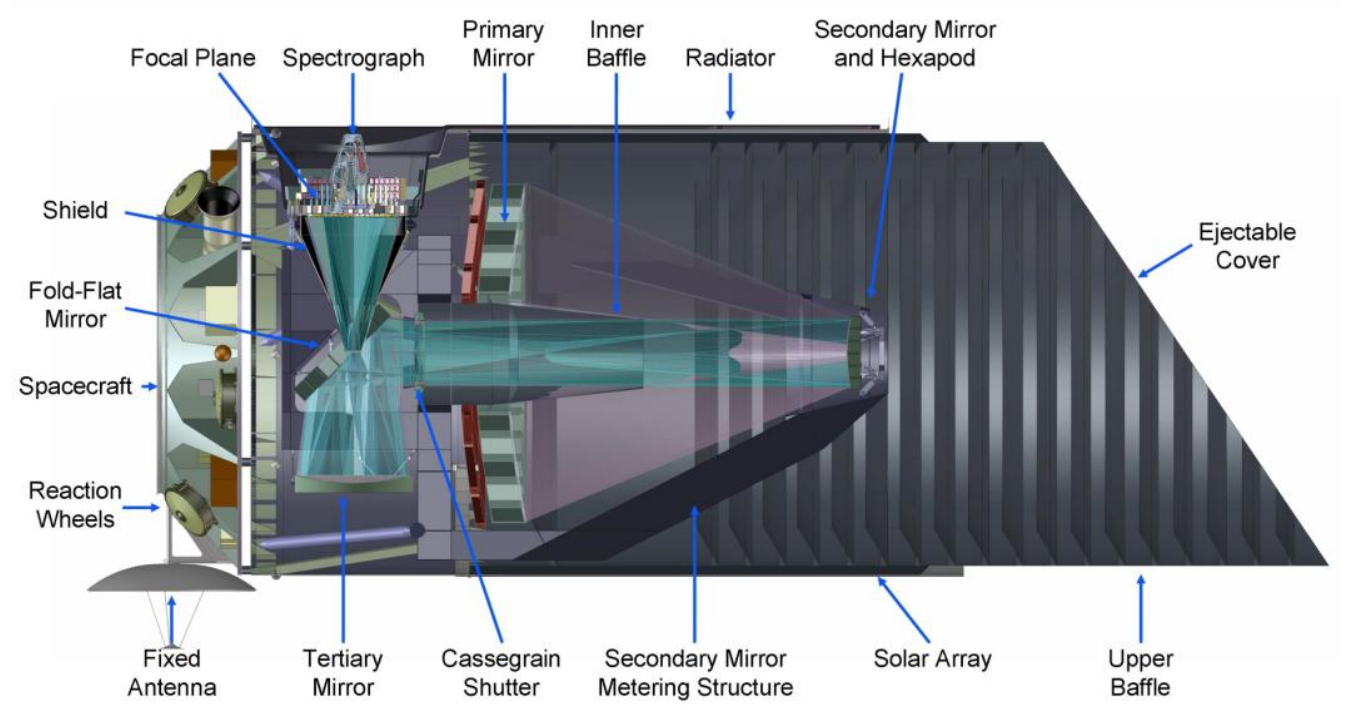

Figure 1. A cross-sectional view of an earlier version of SNAP proposed for JDEM, which shows the TMA design. The focal plane is top left and the light shield is just below that. The ROF would be placed at the top of the shield cone.

There are significant advantages to the ROF. First, high frequency flat fielding can be done routinely and quickly. Second, the ROF can incorporate lamp sources with well-calibrated irradiance that are monitored by NIST-calibrated photodiodes. The ROF can thus provide an accurate absolute flux scale for photometry. Third, the calibrated irradiance on the focal plane from the ROF provides a means to monitor variations in the filters, as well as standard tests of detector performance.

The ROF does have a disadvantage when compared with standard flat fielding techniques in that it doesn't deliver light through the entire optical train. However changes in the flat field due to the optics upstream from the ROF should be seen over large-scales and therefore corrected with star flats and super flats.

\subsection{The Ring of Fire}

The ROF illumination system consists of a focusing mirror, an annular strip of Spectralon [4], a broad-band light source and a set of fiber optics to transmit the light from the source to the ROF. Seven fiber bundles, fixed to the ROF at the top 
of the light shield, carry the light from the source, illuminating the mirror, which then reflects the light onto the Spectralon. Since Spectralon bi-reflectance has a nearly $2 \pi$ distribution, light will be scattered onto the focal plane in an azimuthally symmetric pattern. The choice of location and the ring shape was made so that light to the focal plane would remain unobstructed, thus minimizing system complexity [5]. Figure 2 illustrates the ROF position at the end of light shield as well as the ROF individual components

Sholl [2] performed a study of the uniformity of light incident on the focal plane for the SNAP illumination system. The results of these calculations for a baseline design of seven equally spaced illumination ports, each with an opening angle of 15 degrees, show that the illumination pattern is azimuthally uniform but radially dependent on the distance from the SNAP optical axis. On small pixel scales the uniformity is better than $1 \%$. Figure 3 illustrates the ROF illumination light rays from a single fiber. Light rays are transmitted from the fiber tip, off the focusing mirror, to the Spectralon and then onto the focal plane.
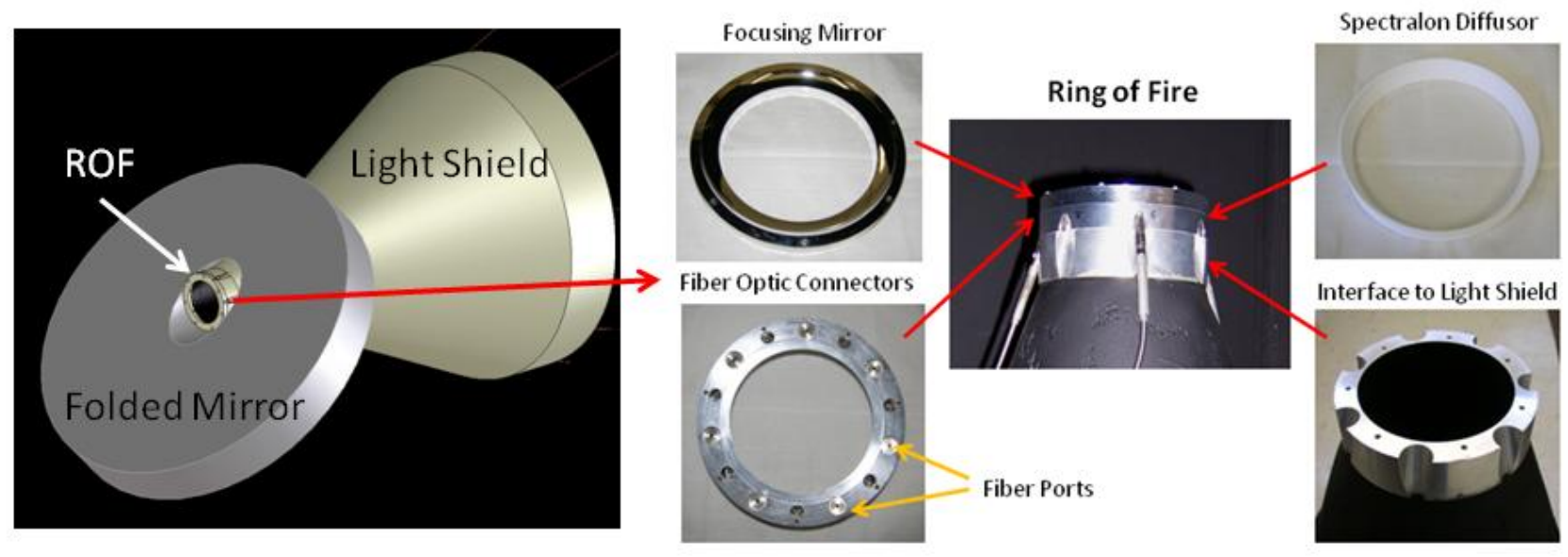

Figure 2. Left illustrates the location of the ROF relative to the light shield and folded mirror in a TMA telescope design. Right illustrates the ROF and its components.

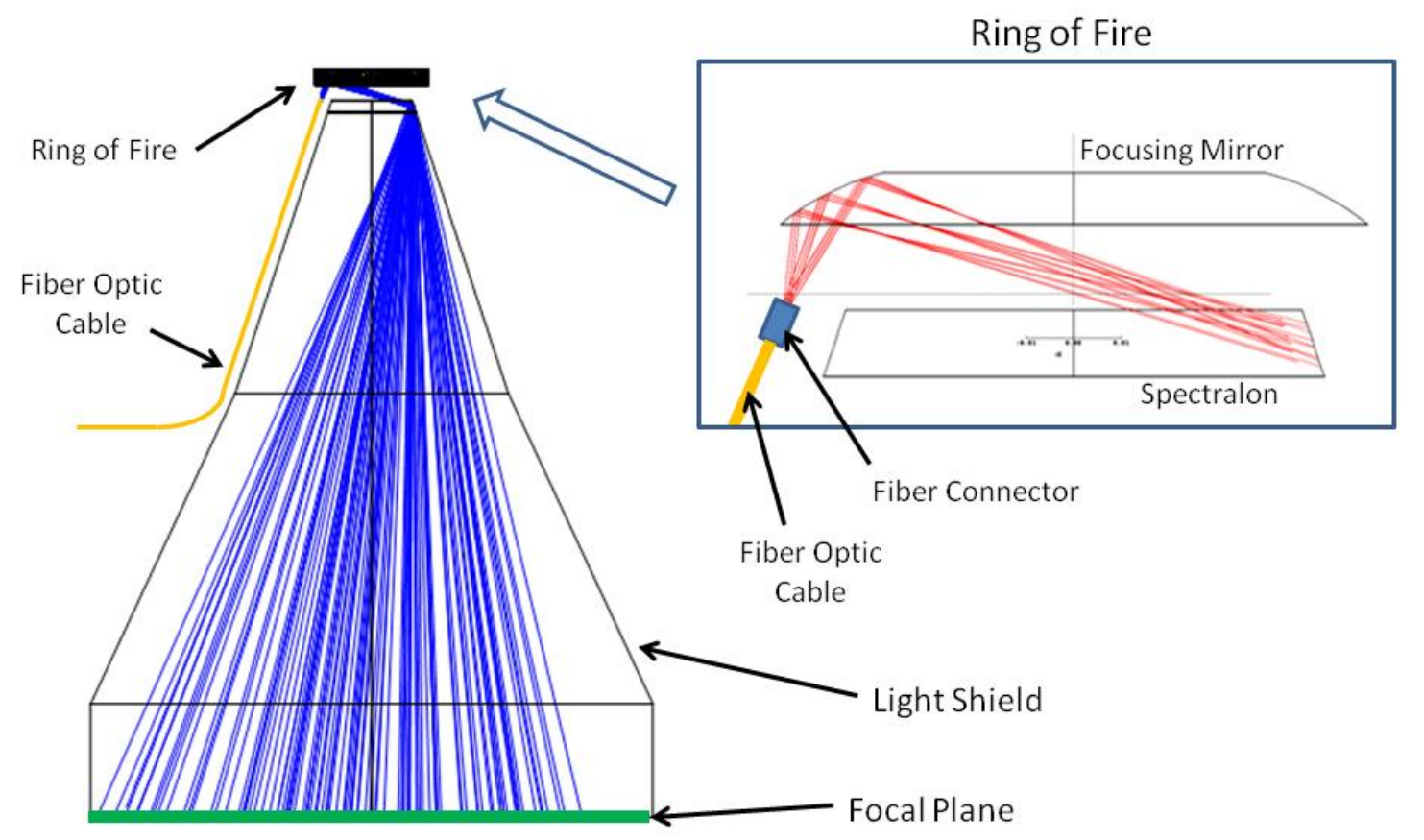

Figure 3. The ROF illumination light rays from a single fiber. Light rays are transmitted from the fiber tip, off the focusing mirror, to the Spectralon and then onto the focal plane. 


\section{RING OF FIRE EXPERIMENTAL SETUP}

A mockup of the ROF illumination system was tested at the Fermi National Accelerator Laboratory. The test setup consists of a illumination source, a dark box, the ROF, fiber optic cables, the light shield and a scanning photodiode assembly. We used a broad-band quartz tungsten halogen (QTH) lamp (Newport, model 6333) as our illumination source. Each fiber optic cable consists of seven individual broad-band, low-OH optical fibers (Polymicro, model STUD), with NASA specified connectors (Diamond, modified AVIM), and were assembled by NASA Goddard Space Flight Center. Because of cost restrictions, only three fiber optic cable assemblies were made for this test. A fiberglass light shield, similar to an early SNAP version, was constructed by Indiana University and painted with Aeroglase Z306 flatblack paint to minimize scattered light effects. The flat field illumination measurements were made with a UV-enhanced silicon photodiode (Newport, model 71640) that was scanned across the focal plane with a x-y stage. The photodiode current was measured with a picoammeter (Keithley, model 6485). Figure 4 shows the ROF test setup.

Because we did not have all seven fiber optic cables available for these measurements, the full ROF illumination pattern was made with a series of single-fiber illuminations measurements. Looking upward from the focal plane, through the light shield toward the ROF, figure 5 shows the single fiber illuminations of the ROF Spectralon for each of the seven fiber ports. The lower-right image in figure 5 shows the Spectralon illumination for all seven fiber ports. This image is constructed from combining the seven individual fiber illumination images. This image shows that the ROF generates a fairly even illumination source around the optical exit pupil.

(a)

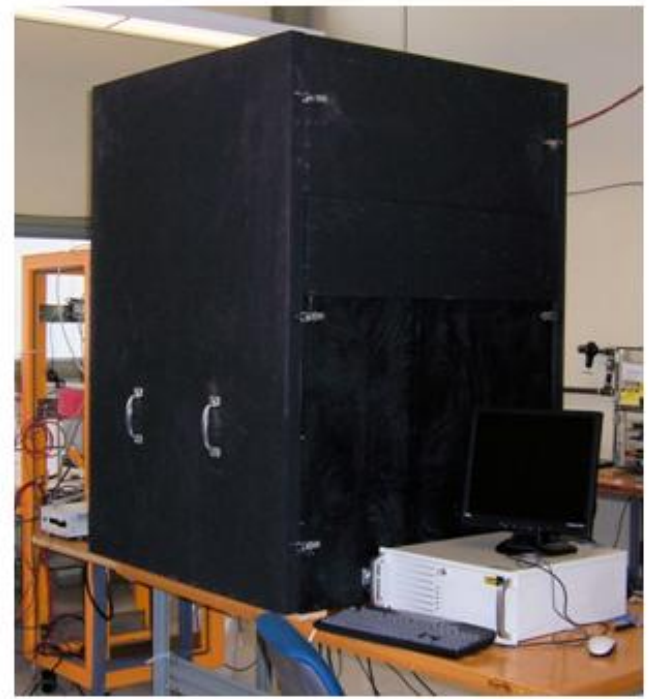

(c)

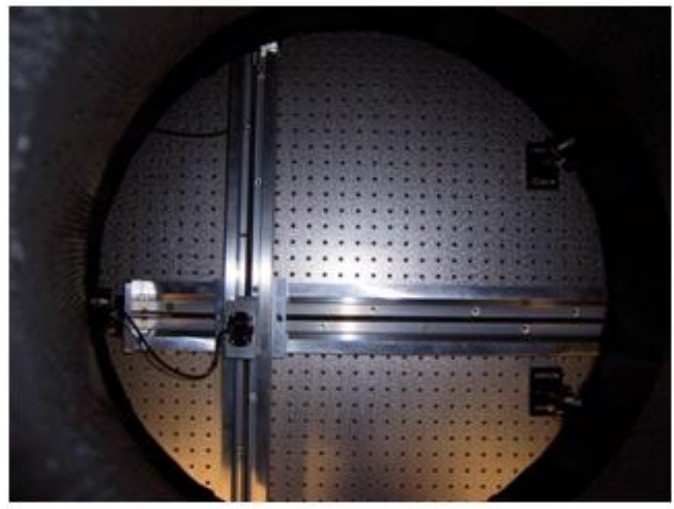

(b)

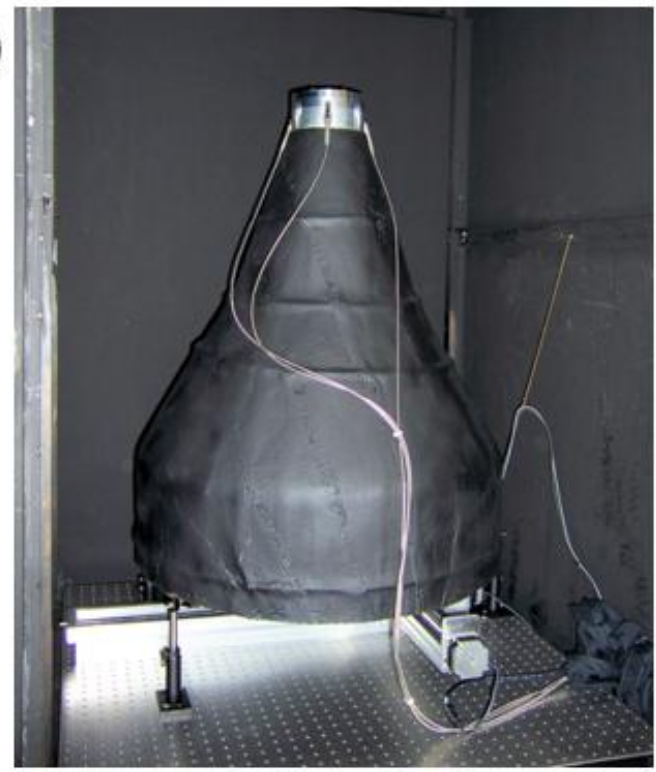

(d)

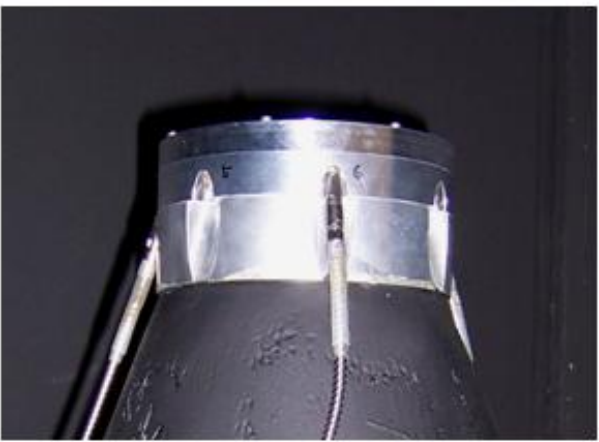

Figure 4. The ROF measurement test setup. (a) Shows the dark box test platform. (b) Shows the ROF, with three fiber optics connected, atop the light shield inside the dark box. (c) Is looking down from the ROF opening toward the scanning photodiode. (d) Shows a close-up of the ROF with fiber optics. 


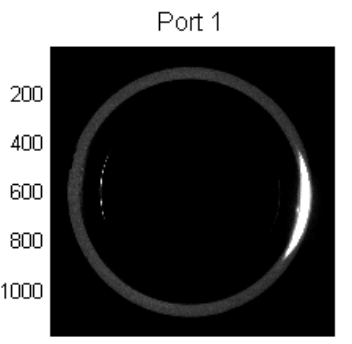

2004006008001000

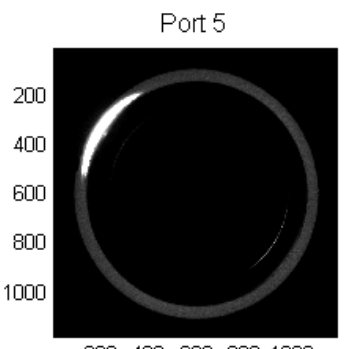

2004006008001000

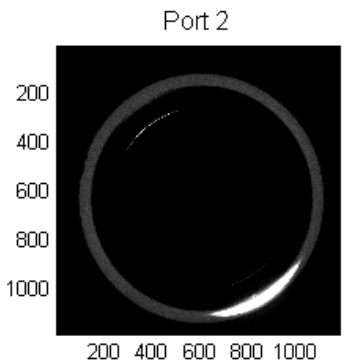

Port 6

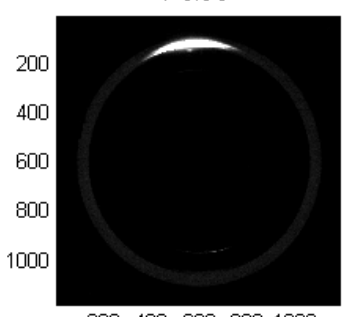

2004006008001000

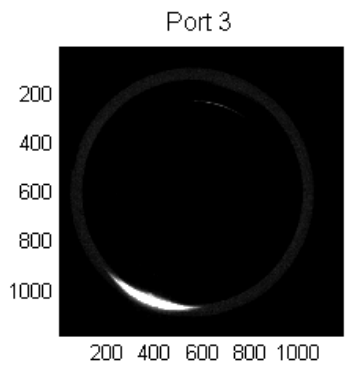

Port 7

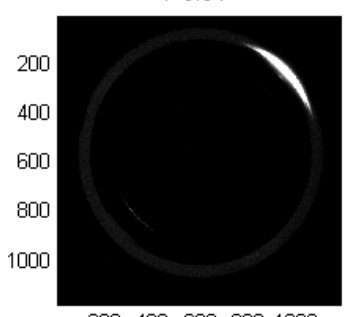

$200400 \quad 6008001000$
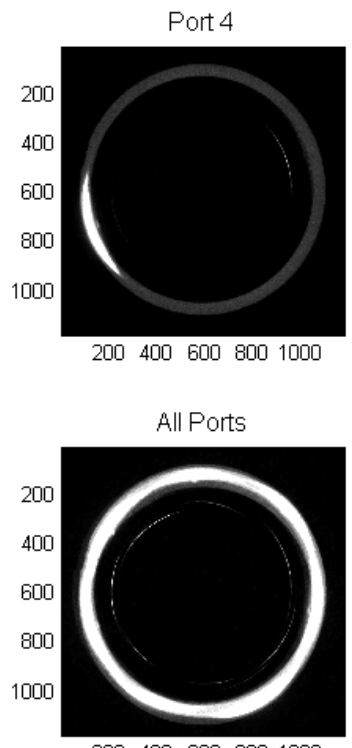

2004006008001000

Figure 5. Looking upward from the focal plane, through the light shield toward the ROF, these images show the single fiber illuminations of the ROF Spectralon. The lower-right image shows the Spectralon illumination for all seven fiber ports. This image is constructed from combining the seven individual fiber illumination images.

\section{RING OF FIRE EXPERIMENTAL MEASUREMENTS}

As mentioned above, the full ROF illumination pattern was made with a series of single-fiber illuminations measurements. Figure 6 shows a series of false-color images of the focal-plane illumination intensity patterns for each of the seven ROF fiber optic ports. The lower right image in figure 6 shows the illumination pattern for all seven fiber ports of the ROF. The outer round shape of these patterns is due to a cut-off in illumination from the edge of the light shield. Figure $7 \mathrm{a}$ shows false-color intensity surface plot for seven fiber illumination of the ROF while figures $7 \mathrm{~b}$ and $7 \mathrm{c}$ show the $\mathrm{X}$ and $\mathrm{Y}$ intensity profiles, respectfully, through peak intensity of figure 7a. The ROF illumination appears peaked in the center of the focal plane at the optical axis and falls off smoothly in the radial direction.
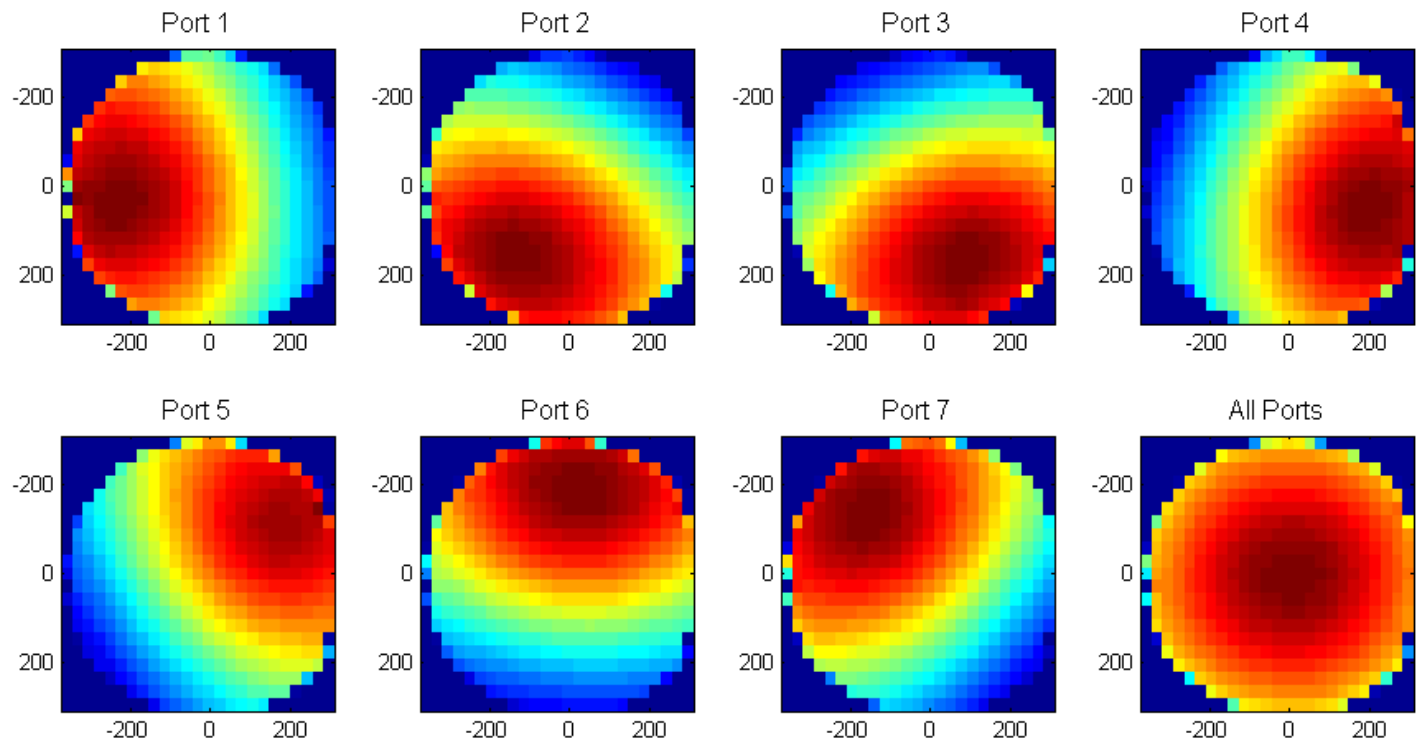

Figure 6. False-color intensity plots from the focal-plane scanning photodiode for single fiber illuminations of the ROF. The lower-right image is sum of all the single fiber intensities. 
(a)

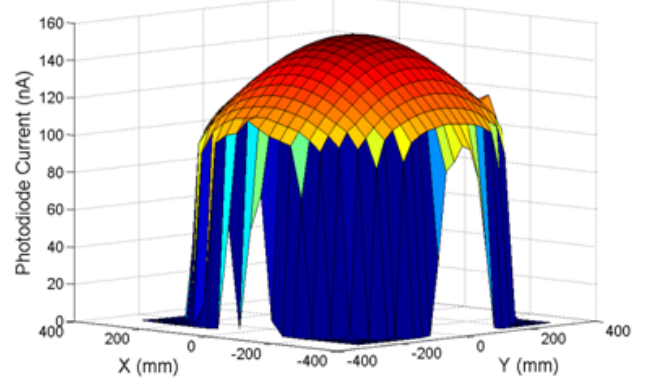

(b)

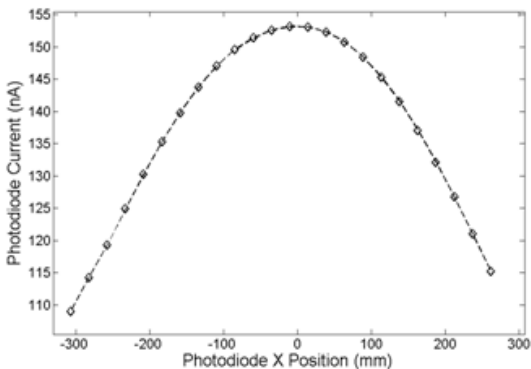

(c)

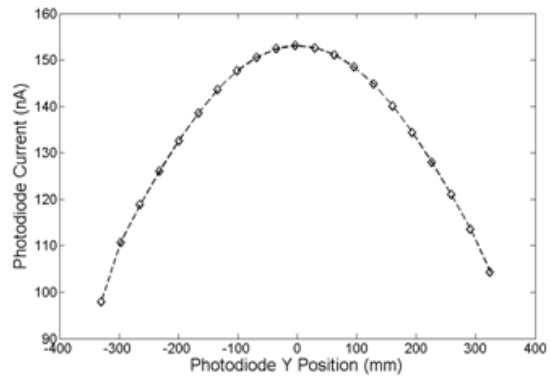

Figure 7. (a) False-color intensity surface plot from the focal-plane scanning photodiode for seven fiber illumination of the ROF. (b) Intensity profile along X scan direction through peak intensity. (c) Intensity profile along Y scan direction through peak intensity.

\section{MEASUREMENTS VS SIMULATION}

A simple model has been constructed to predict the ROF illumination pattern. The model incorporates the basic geometry of the ROF along with simplified treatment of the illumination system. Each fiber is presumed to illuminate the Spectralon at a single point with an incidence angle of 38 degrees. The model propagates the light to the photodiode detector, applying the usual geometric projection factors. The model does include the bidirectional reflectance factor for Spectralon based on data obtained in conjunction with the NASA MISR mission [6].

Figure 8 compares the observed illumination map, obtained by summing the seven single-port illumination maps, with the model prediction. Figure 9 show the $\mathrm{X}$ and $\mathrm{Y}$ profiles taken through the centers of the two maps. The model predicts that the illumination pattern should be dome-shaped, as is observed, but also predicts a shallower decline away from the center than is observed. The reason for this discrepancy is being investigated.
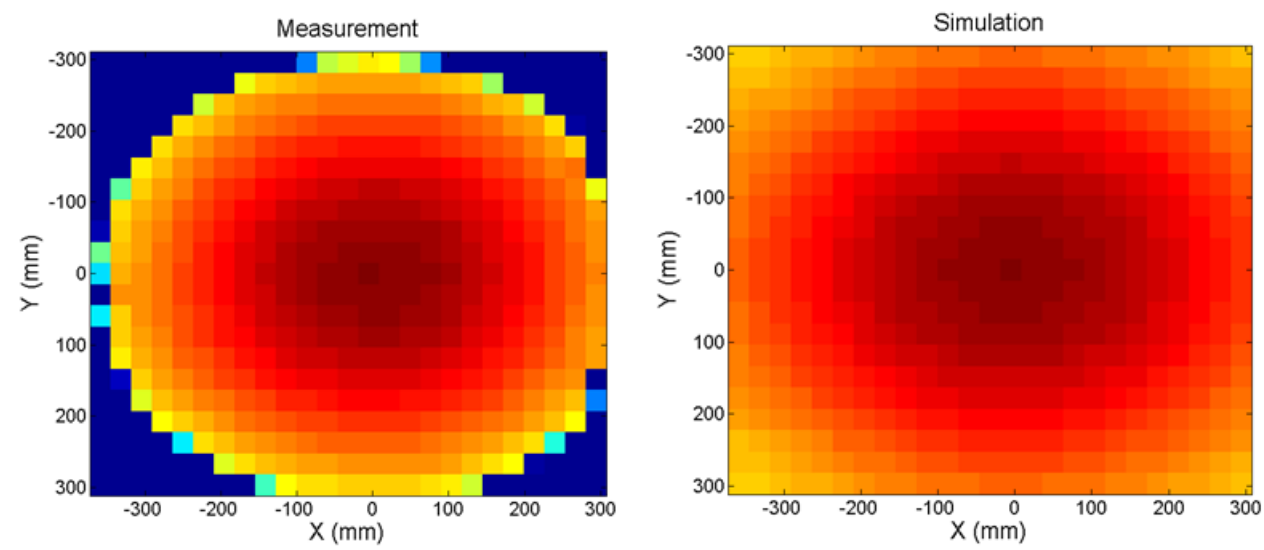

Figure 8 . The left image shows the measured focal plane intensity pattern while the right image show the results from simulation. Both images are normalized to the same peak intensity and the simulation data does not show the edges produced by the light shield. 

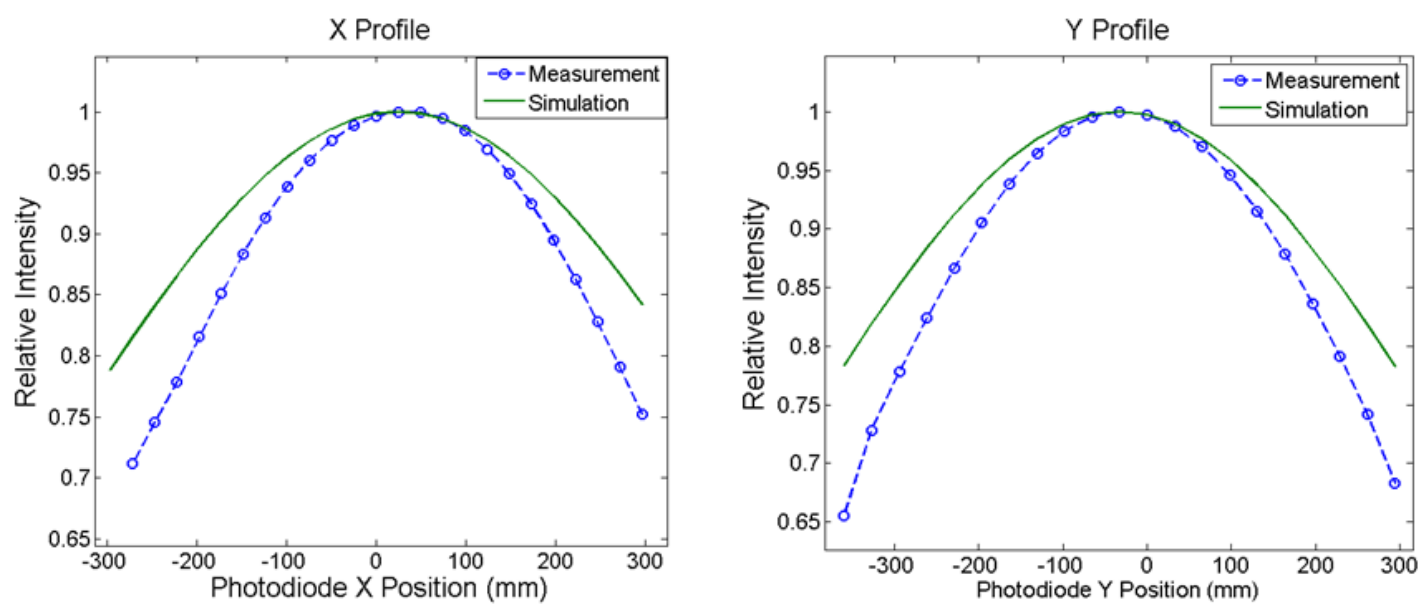

Figure 9. $\mathrm{X}$ and $\mathrm{Y}$ profiles of the images in figure 8 comparing measured intensity to simulation. The profiles are through the peak intensities.

\section{CONCLUSIONS}

This paper describes a prototype of an illumination system, the Ring of Fire, which is part of an internal calibration system for large focal plane detector arrays in TMA telescope designs. The ROF is intended to create high spatial frequency flat fields and to monitor filter bandpasses for experiments that demand a highly accurate characterization of the detectors. Measurements of the focal plane illumination pattern from a broadband light source are presented. The ROF produces a dome-shaped illumination pattern but this pattern does not match the predicted simulation pattern. We are investigating this discrepancy.

\section{REFERENCES}

[1] jdem.gsfc.nasa.gov

[2] Sholl M.J., "SNAP Stray Light Monitoring Status,” SNAP Internal Note (2004).

[3] Bohlin, R., Linder, D., and Riess, A., "Grism Sensitivities and Apparent Non-Linearities," Space Telescope Science Institute, Instrument Science Report NICMOC 2005-002 (2005).

[4] www.labsphere.com

[5] Mostek, N., "Calibration System Design and Determination of Filter Calibration Requirements for SNAP," Ph.D. thesis, Indiana University (2007).

[6] http://www-misr.jpl.nasa.gov/mission/valwork/cal_data/brf/BRF database.dat 